\title{
INEQUALITIES OF THE COMPLETE ELLIPTIC INTEGRALS
}

\author{
FENG QI AND ZHENG HUANG
}

\begin{abstract}
In this article, using Tchebycheff's integral inequality, the authors establish some estimates and inequalities for three kinds of the complete elliptic integrals.
\end{abstract}

\section{Introduction}

It is well-known that the elliptic integrals can not be represented by elementary functions, it is also called as Abel's integral. The complete elliptic integrals are classed into three kinds, they are defined as and denoted by

$$
\begin{aligned}
E(k) & =\int_{0}^{\pi / 2} \sqrt{1-k^{2} \sin ^{2} \theta} d \theta \\
F(k) & =\int_{0}^{\pi / 2} \frac{d \theta}{\sqrt{1-k^{2} \sin ^{2} \theta}} \\
I I(k, h) & =\int_{0}^{\pi / 2} \frac{d \theta}{\left(1+h \sin ^{2} \theta\right) \sqrt{1-k^{2} \sin ^{2} \theta}}
\end{aligned}
$$

where $0<k<1$.

The first kind and second kind of the complete elliptic integral can be expanded into power-series as follows,

$$
\begin{aligned}
& E(k)=\frac{\pi}{2}\left\{1-\sum_{n=1}^{\infty} \frac{(2 n-2) !(2 n) !}{2^{4 n-1}(n-1) !(n !)^{3}} \cdot k^{2 n}\right\}, \\
& F(k)=\frac{\pi}{2} \sum_{n=0}^{\infty} \frac{[(2 n) !]^{2}}{2^{4 n}(n !)^{4}} \cdot k^{2 n} .
\end{aligned}
$$

The first kind of the complete elliptic integral was estimated in $[2,3]$ by

$$
\frac{\pi(a+b)}{4} \leq \int_{0}^{\pi / 2} \sqrt{a^{2} \cos ^{2} \theta+b^{2} \sin ^{2} \theta} d \theta \leq \frac{\pi \sqrt{2\left(a^{2}+b^{2}\right)}}{4},
$$

Received February 21, 1997.

1991 Mathematics Subject Classification. Primary 26D15, 33E05; Secondary 33C55.

Key words and phrases. Inequality, estimate complete elliptic integral, Tchebycheff's integral inequality.

The first author was supported in part by NSF grant no. 974050400 of Henan Province, The People's Republic of China. 


$$
\int_{0}^{\pi / 2} \sqrt{1-k^{2} \sin ^{2} \theta} d \theta \geq \frac{\pi\left(2 a^{2}+b^{2}\right)}{3 a}
$$

where $0<k<1, k=\sqrt{a^{2}-b^{2}} / a$.

Recently, the upper bound of (6) was improved in [1] for $a \geq 7 b$ by a better constant $(a+2 b) \pi / 6$.

Another inequality for the complete elliptic integral was estimated in $[2,12]$ by the following inequality

$$
\frac{\pi}{6}<\int_{0}^{1}\left(4-x^{2}-x^{3}\right)^{-1 / 2} d x<\frac{\pi \sqrt{2}}{8}
$$

The first author improved (8) in $[9,10]$ and got

$$
\frac{3}{10}+\frac{27 \sqrt{2}}{160}<\int_{0}^{1}\left(4-x^{2}-x^{3}\right)^{-1 / 2} d x<\frac{79}{192}+\frac{\sqrt{2}}{10}
$$

In this article, by using Tchebycheff's integral inequality, we get some estimates and inequalities of three kinds of the complete elliptic integrals. The main results are as follows

$$
\begin{gathered}
\frac{\pi \arcsin k}{2 k}<F(k)<\frac{\pi \ln \left(\frac{1+k}{1-k}\right)}{4 k} ; \\
E(k)<\frac{16-4 k^{2}-3 k^{4}}{4\left(4+k^{2}\right)} F(\dot{k}) ; \\
F(k)<\left(1+\frac{h}{2}\right) I I(k, h), \quad-1<h<0, \quad \text { or } \quad h>\frac{k^{2}}{2-3 k^{2}}>0 ; \\
I I(k, h) \cdot E(k)>\frac{\pi^{2}}{4 \sqrt{1+h}},-2<2 h<k^{2} ; \\
E(k) \geq \frac{16-28 k^{2}+9 k^{4}}{4\left(4-5 k^{2}\right)} F(k), \quad k^{2} \leq \frac{2}{3} .
\end{gathered}
$$

For $0<2 h<k^{2}$, the inequality (12) is reversed; for $h>k^{2} /\left(2-3 k^{2}\right)>0$, inequality (13) is reversed.

For our own conveniences, we state the Tchebycheff's integral inequality in $[2,4,5]$, which we will use throughout this article repeatedly, as follows

Lemma. Let $f, g:[a, b] \rightarrow R$ be integrable functions, both increasing or both decreasing. Furthermore, let $p:[a, b] \rightarrow R$ be a positive, integrable function. Then

$$
\int_{a}^{b} p(x) f(x) d x \int_{a}^{b} p(x) g(x) d x \leq \int_{a}^{b} p(x) d x \int_{a}^{b} p(x) f(x) g(x) d x .
$$

If one of the functions $f$ or $g$ is nonincreasing and the other nondecreasing, then the inequality in (15) is reversed. 


\section{Proofs of Inequalities for Elliptic Integrals}

2.1. Let $p(x)=1, f(x)=\left(1-k^{2} \sin ^{2} x\right)^{-1 / 2}, g(x)=\cos x$ or $\sin x,[a, b]=[0, \pi / 2]$ in (15), then we could get

$$
\int_{0}^{\pi / 2} \frac{d x}{\sqrt{1-k^{2} \sin ^{2} x}} \int_{0}^{\pi / 2} \cos x d x>\int_{0}^{\pi / 2} d x \int_{0}^{\pi / 2} \frac{\cos x d x}{\sqrt{1-k^{2} \sin ^{2} x}}
$$

or

$$
\int_{0}^{\pi / 2} \frac{d x}{\sqrt{1-k^{2} \sin ^{2} x}} \int_{0}^{\pi / 2} \sin x d x<\int_{0}^{\pi / 2} d x \int_{0}^{\pi / 2} \frac{\sin x d x}{\sqrt{1-k^{2} \sin ^{2} x}}
$$

By direct calculation one gets the inequality (10), which is the estimates of the second kind of the complete elliptic integral $F(k)$.

2.2. Letting

$$
\begin{aligned}
& p(x)=\left(1+k^{2} \sin ^{2} x / 2\right)\left(1-k^{2} \sin ^{2} x\right), \\
& g(x)=\left(1-k^{2} \sin ^{2} x\right)^{-1}, \\
& f(x)=\left[\left(1+k^{2} \sin ^{2} x / 2\right) \sqrt{1-k^{2} \sin ^{2} x}\right]^{-1},
\end{aligned}
$$

for $x \in[0, \pi / 2]$ in $(15)$, then

$$
\begin{aligned}
& \int_{0}^{\pi / 2} \sqrt{1-k^{2} \sin ^{2} x} d x \int_{0}^{\pi / 2}\left(1+k^{2} \sin ^{2} x / 2\right) d x \\
< & \int_{0}^{\pi / 2}\left(1+k^{2} \sin ^{2} x / 2\right)\left(1-k^{2} \sin ^{2} x\right) d x \int_{0}^{\pi / 2}\left(1-k^{2} \sin ^{2} x\right)^{-1 / 2} d x
\end{aligned}
$$

By direct computation one can obtain inequality (11).

2.3. Set $g(x)=\left(1+h \sin ^{2} x\right) \sqrt{1-k^{2} \sin ^{2} x}, x \in[0, \pi / 2]$, then

$$
g^{\prime}(x)=\frac{\sin x \cos x\left[\left(2 h-k^{2}\right)-3 h k^{2} \sin ^{2} x\right]}{\sqrt{1-k^{2} \sin ^{2} x}}
$$

while $0<2 h<k^{2}$, we have $g^{\prime}(x)<0, g(x)$ decreases; while $h>k^{2} /\left(2-3 k^{2}\right)>0$, we have $g^{\prime}(x)>0, g(x)$ increases; while $-1<h<0$, it is clear that $g(x)$ decreases.

If $-1<h<0$, or $h>k^{2} /\left(2-3 k^{2}\right)>0$, let

$$
p(x)=\left(1-k^{2} \sin ^{2} x\right)^{-1 / 2}, \quad f(x)=\left(1+h \sin ^{2} x\right)^{-1}
$$

and $[a, b]=[0, \pi / 2]$, from $(15)$ one has

$$
I I(k, h) \int_{0}^{\pi / 2}\left(1+h \sin ^{2} x\right) d x>F(k) \int_{0}^{\pi / 2} d x
$$


As a result, inequality (12) holds. If $0<2 h<k^{2}$, inequality (12) is reversed.

2.4. Assume

$$
f(x)=\sqrt{1-k^{2} \sin ^{2} x}, \quad g(x)=\left[\left(1+h \sin ^{2} x\right) \sqrt{1-k^{2} \sin ^{2} x}\right]^{-1},
$$

and $p(x)=1, x \in[0, \pi / 2]$ in (15). For $-2<2 h<k^{2}$ we obtain

$$
I I(k, h) \cdot F(k)>\frac{\pi}{2} \int_{0}^{\pi / 2} \frac{d \theta}{1+h \sin ^{2} \theta}=\frac{\pi^{2}}{4 \sqrt{1+h}} .
$$

For $h>k^{2} /\left(2-3 k^{2}\right)>0$, since $g(x)$ is increasing, the reversed inequality of (13) is obtained.

2.5. For $h=k^{2} /\left(2-3 k^{2}\right)>0$, let

$$
\begin{aligned}
& p(x)=\left(1+h \sin ^{2} x\right)\left(1-k^{2} \sin ^{2} x\right), \\
& f(x)=\left[\left(1+h \sin ^{2} x\right) \sqrt{1-k^{2} \sin ^{2} x}\right]^{-1}, \\
& g(x)=\left(1-k^{2} \sin ^{2} x\right)^{-1}
\end{aligned}
$$

for $x \in[0, \pi / 2]$, the inequality (15) implies (14).

Remark 1. As concrete examples we have the following estimates of the complete elliptic integrals

$$
\begin{gathered}
\frac{\pi^{2}}{4 \sqrt{2}}<\int_{0}^{\pi / 2}\left(1-\frac{\sin ^{2} x}{2}\right)^{-1 / 2} d x<\frac{\pi \ln (1+\sqrt{2})}{\sqrt{2}} \\
\int_{0}^{\pi / 2}\left(1+\frac{\cos x}{2}\right)^{-1} d x<\frac{\pi(\ln 3-\ln 2)}{2} \\
\int_{0}^{\pi / 2}\left(1-\frac{\sin x}{2}\right)^{-1} d x=\int_{\pi / 2}^{\pi}\left(1+\frac{\cos x}{2}\right)^{-1} d x>\frac{\pi \ln 2}{2} .
\end{gathered}
$$

These results are better than those in [2, p. 607].

Remark 2. Using Tchebycheff's integral inequality, we can refine Conte's inequality and some other inequalities relating to the incomplete gamma and the probability functions [8], obtain more particular inequalities [8], and verify the monotonicities of the generalized weighted mean values $[6,7,11]$.

Remark 3. It is clear that, by the similar arguments, we can get inequalities of the incomplete elliptic integrals. 


\section{References}

[1] Bai-Ni Guo and Feng Qi, "Improvemnet for the upper bound of a class of elliptic integral," Journal of Jiaozuo Mining Institute, 14(6)(1995), 125-128. (Chinese)

[2] Ji-Chang Kuang, Applied Inequalities, 2nd edition, Hunan Education Press, Changsha, China, 1993. (Chinese)

[3] Zi-Fen Lu, Gaodeng Shuxue Jiexi Daqun, Liaoning Press of Science and Technology, Shenyang, China, 1991. (Chinese)

[4] D. S. Mitrinović, Analytic Inequalities, Springer-Verlag, 1970.

[5] D. S. Mitrinović, J. E. Pečarić and A. M. Fink, "Classical and New Inequalities in Analysis," Kluwer Academic Publishers, Dordrecht/Boston/London, 1993.

[6] Feng Qi, "Generalized weighted mean values with two parameters," Proc. Roy. Soc. London $A, 454(1998)$, in the press.

[7] Feng Qi, "On a two-parameter family of nonhomogeneous mean values," Tamkang Journal of Mathematics, 29(2)(1998), 83-91.

[8] Feng Qi, Li-Hong Cui and Sen-Lin Xu, "Applications of Tehebycheff's integral inequality," Publ. Inst. Mat. Beograd. N. S. (1998), to appear.

[9] Feng Qi and Bai-Ni Guo, "The estimation of inferior bound for an ellipse integral," Journal of Mathematics for Technology, 10(1)(1994), 87-90. (Chinese).

[10] Feng Qi and Bai-Ni Guo, "Estimate for the upper bound of a complete elliptic integral," Mathematics in Practice and Theory, 26(3)(1996), 285-288. (Chinese)

[11] Feng Qi and Sen-Lin Xu, "The function $\left(b^{x}-a^{x}\right) / x$ : Inequalities and properties," Proc. Amer. Math. Soc. (1998), in the press.

[12] T. M. Rassias, R. H. Garstang and O. P. Lossers, "Bounds on an elliptic integral," Amer. Math. Monthly, 94(1987), 556-557.

Department of Mathematics, Jiaozuo Institute of Technology, Jiaozuo City, Henan 454000, The People's Republic of China; E-mail: qifeng@jzit.edu.cn

Department of Mathematics, University of Science and Technology of China, Hefei City, Anhui 230026, The People's Republic of China. 